\title{
Microbiological Assessment of Some Liquid Herbal Medications Sold in Awka Metropolis, Anambra State
}

\author{
Archibong, Etim Joseph", Igboeli, Chinechendo Ngozi, Okoro, Nnenna Catherine, Obika, Ifeanyi
}

Faculty of Biosciences, Nnamdi Azikiwe University, Nigeria

Copyright $\bigcirc 2017$ by authors, all rights reserved. Authors agree that this article remains permanently open access under the terms of the Creative Commons Attribution License 4.0 International License

\begin{abstract}
The aim of this study is to microbiologically assess some liquid herbal medications sold in Awka, Anambra state, Nigeria. Sixty liquid herbal preparations (twenty NAFDAC registered and forty unregistered) were purchased from different vendors in Awka and analyzed for their microbiological quality by determining the levels of bacteria and fungi in them. Aliquots of the various serially diluted herbal products were cultured on various media plates; NA, SDA, MCA, EMB agar and XLD agar. Total bacterial count of $1.0 \times 10^{3}-2.1 \times 10^{6} \mathrm{cfu} / \mathrm{ml}$, total coliform count of $1 \times 10^{3}-7.8 \times 10^{4} \mathrm{cfu} / \mathrm{ml}$; E.coli count of $1.0 \times$ $10^{2}-2.0 \times 10^{2} \mathrm{cfu} / \mathrm{ml}$ and total fungal count of $1.0 \times 10^{2}-1.4 \times$ $10^{5} \mathrm{cfu} / \mathrm{ml}$ were obtained from NAFDAC registered ones while the unregistered ones had a total bacterial count of 3.6 x $10^{3}-1.11 \times 10^{7} \mathrm{cfu} / \mathrm{ml}$; total coliform count of $2.0 \times 10^{2}$ to $4.4 \times 10^{5} \mathrm{cfu} / \mathrm{ml} ; E$. coli count of $1.0 \times 10^{2}-7.0 \times 10^{4} \mathrm{cfu} / \mathrm{ml}$ and a total fungal count of $2.0 \times 10^{2}$ to $2.0 \times 10^{6} \mathrm{cfu} / \mathrm{ml}$. The result showed that seventy percent of the registered drugs met the WHO standard for liquid herbal drugs while twenty-eight percent of the unregistered ones met the standard. There was a significant difference $(\mathrm{P}<0.05)$ between the total heterotrophic bacterial count and total coliform of registered and unregistered drugs and insignificant differences $(\mathrm{P}>0.05)$ between their total fungal count and E. coli count. Isolates gotten from both samples includes Escherichia coli (21.6\%), Enterobacter asburiae (25\%), Providencia rettgeri (13.3\%), Acinetobacter baumannii (11.6\%), Staphylococcus sp (16.6\%), Bacillus sp (10\%), Scedosporium aurantiacum (5\%), Candida albicans (28.3\%) and Candida krusei (13.3\%). The antibiotic susceptibility test showed that $E$. coli was resistant to most of the antibacterial drugs used and Acinetobacter baumannii was the most susceptible while Penicillium marneffei was the most resistant to the antifungal drugs used and Phialophora parasiticum was the most susceptible. The research work showed that the microbial quality of these herbal drugs is low compared to standard of World Health Organization and could pose a great health risk to the society.
\end{abstract}

Keywords Herbal Preparations, Antibacterial Drugs, Bacterial Count, Fungal Count, Microbial

\section{Introduction}

The use of herbal treatment is an old practice usually employed in diseases treatments. It is sometimes referred to as complementary and alternative medicine (CAM). It is becoming more mainstream as improvements in analysis and quality control along with advances in clinical research show the value of herbal medicine in the treating and preventing diseases. The World Health Organization (WHO) estimates that four billion people (about 80 percent of the world population) use herbal medicine for some aspect of primary health care [1]. The term "herbal drugs" denotes plants and plant parts that have been converted into phytopharmaceuticals by means of some simple processes involving harvesting, drying and storage [2]. According to the World Health Organization, herbal preparations contain plant parts or plant material in crude or processed state as active ingredients and may contain excipients (foreign substances) [3]. It is important to note that any combination with chemically defined active substances or isolated constituents is not considered herbal preparations [1]. Similarly, the European Medicine Evaluation Agency (EMEA) defines herbal preparations as medicinal product containing exclusively herbal drugs or herbal drug preparations as active substances [2].

Herbal medicine has been known to be used to treat many conditions, such as asthma, eczema, irritable bowel syndrome, premenstrual syndrome, rheumatoid arthritis, migraine, menopausal symptoms, chronic fatigue and cancer among others [4]. Widespread use of herbal medicines, calls for the assurance of sustainable availability of quality and safe herbal medicines to ensure continued access especially for rural communities, without compromising patients safety [5]. Interest in medicinal plants as a re-emerging health aid has been fuelled by the rising costs of prescription drugs in the maintenance of personal health and wellbeing. In Nigeria, even though, there is proliferation of herbal products in the market, not so much has been done in this field. Some 
producers of herbal preparations in Nigeria do not have the required expertise to perform quality control on the preparation they produce. This brings about the problem of inconsistency on the quality of the herbal preparation in the country.

With the ever increasing use of herbal medicines and the global expansion of the medicines market safety has become a concern for both health authorities and the public in many counties. This is because many contaminants and residues that may cause harm to the consumers have been reported [6]. The quality assessment of herbal medications is therefore very important in order to justify their acceptance in modern system of medicines. It is thus necessary that the microbiological limit tests of herbal medicinal preparations be done to ensure that the product is free from risk. Some of the formulations are not subject to aseptic conditions during various stages of preparation, packaging, storage and transportation, as required by regulatory norms of NAFDAC (National Agency for Food and Drug Administration and Control) which aim at achieving high standards of quality of food and drugs, including herbal preparations in Nigeria. Plants and plant materials also carry huge number of organisms mainly originating from the soil. Aerobic sporulating bacteria frequently predominate in this to which additional contamination and microbial growth occur during harvesting, handling and production [7]. It is important that physicians and herbalists have the knowledge about the microbiological safety of these preparations [8].

\section{Materials and Methods}

\section{Sample Collection}

Sixty (60) different brands of liquid herbal medications (twenty NAFDAC registered ones and forty unregistered ones) were purchased from different sellers in Awka, Anambra State. The packaged herbal mixtures and the unpackaged (such as those herbal preparations sold by local herbalists) were collected in sterile sampling bottles and taken to microbiology laboratory in Nnamdi Azikiwe University, Awka for analysis.

\section{Microbial Analysis}

The sealed bottles of herbal preparations were cleaned with $70 \%$ ethanol before opening to prevent contamination. Microbial quality was determined by standard plate count method as described by [9]. Total bacterial count, total fungal count, total coliform count and Salmonellae count were done. Each liquid preparation was serially diluted to get a ten-fold dilution before being plated out in duplicate using last two concentrations on different media plate to suit the type of organisms being counted. Nutrient agar was used to count for total bacteria, Sabouraud dextrose agar was used for total fungal count, MacConkey agar was used for total coliform count, Eosine Methylene Blue agar was used for fecal indicator E.coli count and Xylose Lysine Desoxycholate agar were used for Salmonellae count For bacterial counts the plates were then incubated at $37^{\circ} \mathrm{C}$ for 24 hours but for the fungal count, the plates were incubated.

\section{Isolation and Identification of Bacterial Isolates}

Pure cultures of bacterial isolates were obtained, characterized and identified based on, colonial morphology, cellular morphology, biochemical characteristics (indole test, methyl red test, Voges-Proskeur tests, citrate utilization test, urease test, catalase test, coagulase test, oxidase test, spore stain test, motility test and sugar fermentation test). The isolates were identified molecularly using 16sRNA at Biotec Company, Pretoria, South Africa.

\section{Isolation and Identification of Fungal Isolates}

Pure cultures of the fungal isolates were obtained and were identified based on yeast and moulds. The yeasts were identified using the following tests: direct mount, wet mount, gram stain, germ tube test, and urease test and sugar assimilation. Moulds were identified using macroscopic characterization, wet mount, slide cultures, microscopic characterization using fungal atlas.

\section{Statistical Analysis}

Analysis of variance(one-way ANOVA) was used to check for significant differences between total bacterial counts, fungal counts, coliform count and fecal indicator count of NAFDAC registered and unregistered herbal drugs.

\section{Antibiotic Susceptibility Test}

Bacteria isolated from the herbal preparations were screened for their susceptibilities to the selected antibiotics, clinical laboratory and standard institute [10] methods were employed. Antimicrobial sensitivity test of bacteria was one using the disc diffusion method on Mueller Hinton Agar. Pure isolates were emulsified in a small volume of peptone water and the turbidity of the suspension was matched against McFarland standard [11]. $0.1 \mathrm{ml}$ of the suspension was spread on the agar plate. Commercial antibiotic discs were aseptically placed on the inoculated plates and incubated at $35^{\circ} \mathrm{C}$ for 24 hours. The zones of inhibition were measured and recorded. The antibiotic discs used were amoxicillin, streptomycin, septrin, chloramphenicol ciprofloxacin, sparfloxacin, perfloxacin, gentamycin, augmentin, amoxil streptomycin, septrin, zinnacef, erythromycin, ampiclox, rifampicin and tarivid. Agar well diffusion method on Mueller Hinton agar was used for antibiotic sensitivity of fungi. Pure cultures of the fungi isolates were emulsified in peptone water and the suspensions were matched against McFarland standard. The $0.1 \mathrm{ml}$ of the standardized suspension was then inoculated on 
the agar plate by spreading on the agar plate, after which wells of $6 \mathrm{~mm}$ was dug on the agar plate. $0.1 \mathrm{ml}$ of different concentrations of antifungal dilution was placed into each well [12]. The antifungal drugs used were clotrimazole, griseofulvin, nystatin, ketoconazole and fluconazole. The plates were then incubated at $25^{\circ} \mathrm{C}$ for five days and the zones of inhibitions measured and recorded.

\section{Results}

Table 1 shows that the microbial counts of the liquid herbal medications which indicates that three $(15 \%)$ of the registered herbal preparations were free from microbial contamination, seven $(35 \%)$ had no bacterial growth, eight $(40 \%)$ had total bacterial count in the range of $1.0 \times 10^{3}$ to 5.9 $\mathrm{x} 10^{3} \mathrm{cfu} / \mathrm{ml}$, theses complied with the WHO limits for bacterial loads [13]. Six (30\%) had total bacterial load range of $1.4 \times 10^{5}$ to $2.1 \times 10^{6} \mathrm{cfu} / \mathrm{ml}$ which does not comply WHO standards. Five $(25 \%)$ had no fungal growth, ten $(50 \%)$ had fungal count between $1.0 \times 10^{2}$ and $1.0 \times 10^{3} \mathrm{cfu} / \mathrm{ml}$ (these complied with the standard) while five (25\%) showed counts between $1.4 \times 10^{3}$ and $1.4 \times 10^{5} \mathrm{cfu} / \mathrm{ml}$ (they did not comply with the standard). Table 2 indicates that twelve (30\%) of the 40 unregistered herbal preparations had bacterial counts in the range of $3.6 \times 10^{3}$ to $1.09 \times 10^{4} \mathrm{cfu} / \mathrm{ml}$ thus complying with the WHO standard. Twenty eight $(70 \%)$ had bacterial counts in the range of $1.13 \times 10^{5}$ to $1.11 \times 10^{7} \mathrm{cfu} / \mathrm{ml}$, these do not comply with the standard. Two $(5 \%)$ had fungal count between $2.0 \times 10^{2}$ and $1.0 \times 10^{3} \mathrm{cfu} / \mathrm{ml}$ (they complied with the standard), thirty eight (95\%) showed fungal count between $2.0 \times 10^{3}$ and $2.0 \times 10^{6} \mathrm{cfu} / \mathrm{ml}$ thus complying with the standards of the WHO.

Table 1. Microbial Counts of the Registered Liquid Herbal Medications (Cfu/ml)

\begin{tabular}{|c|c|c|c|c|c|c|}
\hline $\mathrm{S} / \mathrm{N}$ & Product code & Bacteria & Fungi & Coliforms & E. coli & Salmonella \\
\hline 1 & $\mathrm{AI}$ & $2.1 \times 10^{6}$ & $2.5 \times 10^{3}$ & $7.5 \times 10^{3}$ & $1.0 \times 10^{2}$ & - \\
\hline 2 & SK & $3.7 \times 10^{5}$ & $2.0 \times 10^{4}$ & $3.4 \times 10^{3}$ & $1.0 \times 10^{2}$ & - \\
\hline 3 & $\mathrm{DN}$ & - & $1.0 \times 10^{2}$ & - & - & - \\
\hline 4 & GC & $3.8 \times 10^{3}$ & $1.0 \times 10^{2}$ & $1.0 \times 10^{3}$ & - & - \\
\hline 5 & GJ & - & $2.0 \times 10^{2}$ & - & - & - \\
\hline 6 & $\mathrm{AV}$ & - & - & - & - & - \\
\hline 7 & KA & $1.2 \times 10^{3}$ & $1.0 \times 10^{2}$ & - & - & - \\
\hline 8 & $\mathrm{TL}$ & - & $1.0 \times 10^{2}$ & - & - & - \\
\hline 9 & AS & $1.4 \times 10^{5}$ & - & $3.0 \times 10^{3}$ & $2.0 \times 10^{2}$ & - \\
\hline 10 & $\mathrm{AH}$ & $7.6 \times 10^{5}$ & $1.0 \times 10^{4}$ & $3.6 \times 10^{3}$ & - & - \\
\hline 11 & $\mathrm{AR}$ & - & $1.0 \times 10^{3}$ & - & - & - \\
\hline 12 & $\mathrm{NO}$ & - & - & - & - & - \\
\hline 13 & DR & $1.0 \times 10^{3}$ & $1.0 \times 10^{2}$ & - & - & - \\
\hline 14 & OS & - & - & - & - & - \\
\hline 15 & DA & $5.9 \times 10^{3}$ & - & $1.0 \times 10^{3}$ & $1.0 \times 10^{2}$ & - \\
\hline 16 & $\mathrm{BO}$ & $3.2 \times 10^{5}$ & $1.0 \times 10^{3}$ & - & - & - \\
\hline 17 & $\mathrm{AC}$ & $1.0 \times 10^{3}$ & $1.0 \times 10^{2}$ & - & - & - \\
\hline 18 & BI & $4.2 \times 10^{5}$ & $1.4 \times 10^{3}$ & $1.0 \times 10^{3}$ & - & - \\
\hline 19 & $\mathrm{IM}$ & $1.6 \times 10^{5}$ & $1.4 \times 10^{5}$ & $7.8 \times 10^{4}$ & - & - \\
\hline 20 & $\mathrm{DM}$ & $9.8 \times 10^{5}$ & $2.3 \times 10^{4}$ & $3.2 \times 10^{4}$ & - & - \\
\hline
\end{tabular}

Key: $=$ no count, $* \mathrm{P}>0.05($ ANOVA) 
Table 2. Microbial Count of the Unregistered Liquid Herbal Medications (Cfu/ml)

\begin{tabular}{|c|c|c|c|c|c|c|}
\hline $\mathrm{S} / \mathrm{N}$ & Product code & Bacteria & Fungi & Coliforms & E. coli & Salmonella \\
\hline 1 & $\mathrm{MA}_{1}$ & $3.9 \times 10^{5}$ & $1.0 \times 10^{4}$ & $5.0 \times 10^{3}$ & - & - \\
\hline 2 & $\mathrm{MA}_{2}$ & $4.9 \times 10^{6}$ & $1.0 \times 10^{4}$ & $6.0 \times 10^{3}$ & $1.0 \times 10^{3}$ & - \\
\hline 3 & $\mathrm{MA}_{3}$ & $3.7 \times 10^{6}$ & $1.0 \times 10^{4}$ & - & - & - \\
\hline 4 & $\mathrm{MA}_{4}$ & $7.6 \times 10^{6}$ & $2.0 \times 10^{4}$ & $2.3 \times 10^{4}$ & - & - \\
\hline 5 & $\mathrm{MA}_{5}$ & $1.08 \times 10^{5}$ & $4.0 \times 10^{3}$ & $5.6 \times 10^{4}$ & $6.0 \times 10^{3}$ & - \\
\hline 6 & $\mathrm{SD}_{1}$ & $1.67 \times 10^{5}$ & $3.1 \times 10^{3}$ & $3.5 \times 10^{3}$ & $4.0 \times 10^{2}$ & - \\
\hline 7 & $\mathrm{SD}_{2}$ & $3.3 \times 10^{6}$ & $1.0 \times 10^{4}$ & - & - & - \\
\hline 8 & $\mathrm{SD}_{3}$ & $5.7 \times 10^{6}$ & $1.0 \times 10^{4}$ & $4.2 \times 10^{3}$ & $1.2 \times 10^{2}$ & - \\
\hline 9 & $\mathrm{SD}_{4}$ & $8.2 \times 10^{6}$ & $2.3 \times 10^{3}$ & $6.6 \times 10^{3}$ & - & - \\
\hline 10 & $\mathrm{SD}_{5}$ & $6.0 \times 10^{6}$ & $2.0 \times 10^{3}$ & $1.0 \times 10^{4}$ & - & - \\
\hline 11 & $\mathrm{TP}_{1}$ & $2.37 \times 10^{5}$ & $2.0 \times 10^{3}$ & $5.7 \times 10^{3}$ & $4.0 \times 10^{2}$ & - \\
\hline 12 & $\mathrm{TP}_{2}$ & $1.13 \times 10^{5}$ & $9.8 \times 10^{3}$ & $1.3 \times 10^{3}$ & $1.0 \times 10^{2}$ & - \\
\hline 13 & $\mathrm{TP}_{3}$ & $1.28 \times 10^{6}$ & $5.2 \times 10^{3}$ & $4.2 \times 10^{3}$ & $3.0 \times 102^{2}$ & - \\
\hline 14 & $\mathrm{TP}_{4}$ & $7.7 \times 10^{4}$ & $2.0 \times 10^{2}$ & $1.0 \times 10^{3}$ & - & - \\
\hline 15 & $\mathrm{TP}_{5}$ & $4.1 \times 10^{4}$ & $6.0 \times 10^{2}$ & - & - & - \\
\hline 16 & $\mathrm{MD}_{1}$ & $2.42 \times 10^{8}$ & $3.0 \times 10^{4}$ & $1.0 \times 10^{5}$ & $1.0 \times 10^{4}$ & - \\
\hline 17 & $\mathrm{MD}_{2}$ & $5.7 \times 10^{6}$ & $1.2 \times 10^{5}$ & $1.2 \times 10^{5}$ & $2.2 \times 10^{5}$ & - \\
\hline 18 & $\mathrm{MD}_{3}$ & $2.3 \times 10^{4}$ & $1.0 \times 10^{4}$ & $1.0 \times 10^{5}$ & - & - \\
\hline 19 & $\mathrm{MD}_{4}$ & $3.6 \times 10^{3}$ & $2.0 \times 10^{2}$ & $2.0 \times 10^{2}$ & - & - \\
\hline 20 & $\mathrm{MD}_{5}$ & $1.11 \times 10^{7}$ & $2.0 \times 10^{6}$ & $4.4 \times 10^{5}$ & $7.0 \times 10^{4}$ & - \\
\hline 21 & $\mathrm{BP}_{1}$ & $2.0 \times 10^{6}$ & $1.3 \times 10^{5}$ & $7.0 \times 10^{3}$ & $2.0 \times 10^{2}$ & - \\
\hline 22 & $\mathrm{BP}_{2}$ & $5.0 \times 10^{4}$ & $1.0 \times 10^{4}$ & $1.5 \times 10^{4}$ & - & - \\
\hline 23 & $\mathrm{BP}_{3}$ & $7.5 \times 10^{4}$ & $1.0 \times 10^{3}$ & $2.2 \times 10^{3}$ & $1.0 \times 10^{3}$ & - \\
\hline 24 & $\mathrm{BP}_{4}$ & $1.17 \times 10^{6}$ & $2.0 \times 10^{4}$ & $5.0 \times 10^{4}$ & $1.0 \times 10^{2}$ & - \\
\hline 25 & $\mathrm{BP}_{5}$ & $1.28 \times 10^{5}$ & $4.7 \times 10^{4}$ & $7.2 \times 10^{3}$ & $6.0 \times 10^{2}$ & - \\
\hline 26 & $\mathrm{IF}_{1}$ & $2.19 \times 10^{5}$ & $1.5 \times 10^{3}$ & $3.4 \times 10^{3}$ & - & - \\
\hline 27 & $\mathrm{IF}_{2}$ & $3.7 \times 10^{6}$ & $2.0 \times 10^{5}$ & $2.4 \times 10^{4}$ & $2.0 \times 10^{3}$ & - \\
\hline 28 & $\mathrm{IF}_{3}$ & $9.0 \times 10^{4}$ & $6.2 \times 10^{3}$ & - & - & - \\
\hline 29 & $\mathrm{IF}_{4}$ & $5.1 \times 10^{4}$ & $1.0 \times 10^{3}$ & - & - & - \\
\hline 30 & $\mathrm{IF}_{5}$ & $1.32 \times 10^{5}$ & $6.0 \times 10^{3}$ & $5.8 \times 10^{3}$ & $1.2 \times 10^{3}$ & - \\
\hline 31 & $\mathrm{SM}_{1}$ & $2.08 \times 10^{5}$ & $1.9 \times 10^{4}$ & $1.0 \times 10^{4}$ & - & - \\
\hline 32 & $\mathrm{SM}_{2}$ & $2.19 \times 10^{6}$ & $2.7 \times 10^{4}$ & $7.2 \times 10^{3}$ & $4 \times 10^{2}$ & - \\
\hline 33 & $\mathrm{SM}_{3}$ & $1.78 \times 10^{5}$ & $3.0 \times 10^{5}$ & $6.2 \times 10^{4}$ & - & - \\
\hline 34 & $\mathrm{SM}_{4}$ & $9.2 \times 10^{6}$ & $4.0 \times 10^{5}$ & $4.0 \times 10^{5}$ & - & - \\
\hline 35 & $\mathrm{PL}_{1}$ & $2.72 \times 10^{5}$ & $2.3 \times 10^{4}$ & $7.9 \times 10^{3}$ & - & - \\
\hline 36 & $\mathrm{PL}_{2}$ & $1.0 \times 10^{6}$ & $1.0 \times 10^{4}$ & $2.3 \times 10^{3}$ & $6.0 \times 10^{2}$ & - \\
\hline 37 & $\mathrm{WP}_{1}$ & $1.46 \times 10^{5}$ & $2.9 \times 10^{3}$ & $4.2 \times 10^{3}$ & - & - \\
\hline 38 & $\mathrm{WP}_{2}$ & $1.09 \times 10^{4}$ & $4.4 \times 10^{2}$ & - & - & - \\
\hline 39 & $\mathrm{TA}_{1}$ & $2.12 \times 10^{4}$ & $1.0 \times 10^{3}$ & $1.0 \times 10^{3}$ & - & - \\
\hline 40 & $\mathrm{TA}_{2}$ & $\mathrm{x} 10^{5}$ & $2.2 \times 10^{3}$ & $1.0 \times 10^{4}$ & $1.2 \times 10^{2}$ & - \\
\hline
\end{tabular}

Key: - = no count, $* \mathrm{P}>0.05($ ANOVA) 
Table 3. Morphological and Biochemical Characteristics of Bacterial Isolates from the Liquid Herbal Medications

\begin{tabular}{|c|c|c|c|c|c|c|c|c|c|c|c|c|c|c|c|c|c|}
\hline morphology & $\begin{array}{c}\text { Gram } \\
\text { reaction }\end{array}$ & Indole & $\begin{array}{l}\text { Methyl } \\
\text { red }\end{array}$ & VogesProkeur & Urease & Motility & Citerate & Glucose & Sucrose & Maltose & Raffinose & Mannitol & Lactose & Oxidase & Catalase & Coagulase & $\begin{array}{l}\text { Probable } \\
\text { organism }\end{array}$ \\
\hline Rods & - & + & + & - & + & + & + & $\mathrm{AG}$ & - & - & - & A & A & - & + & & $\begin{array}{c}\text { Providencia } \\
\text { rettgeri }\end{array}$ \\
\hline Rods & - & - & + & - & + & - & + & $\mathrm{AG}$ & A & A & A & A & A & - & - & & $\begin{array}{c}\text { Enterobacter } \\
\text { asburiae }\end{array}$ \\
\hline Rods & - & - & + & + & + & - & + & $\mathrm{AG}$ & AG & A & - & - & - & - & + & & $\begin{array}{c}\text { Acinetobacter } \\
\text { baumanii }\end{array}$ \\
\hline Rods & - & + & + & - & - & + & - & $\mathrm{AG}$ & $\mathrm{AG}$ & A & - & - & $\mathrm{AG}$ & - & + & & $\begin{array}{c}\text { Escherichia } \\
\text { coli }\end{array}$ \\
\hline Rods & + & - & + & - & - & + & + & $\mathrm{AG}$ & - & - & - & - & - & - & + & & Bacillus sp \\
\hline Coccus & + & 0 & 0 & 0 & 0 & 0 & 0 & 0 & 0 & 0 & 0 & 0 & 0 & 0 & + & + & $\begin{array}{c}\text { Staphylococcus } \\
s p\end{array}$ \\
\hline
\end{tabular}

Keys:

$$
\begin{array}{ll}
-= & \text { negative } \\
+ & =\text { positive } \\
\mathrm{A} & =\text { acid } \\
\mathrm{G} & =\text { gas } \\
0 & =\text { no test }
\end{array}
$$


Table 4. Colonial and Microscopic Characteristics of Mould from the Liquid Herbal Medications

\begin{tabular}{|c|c|c|}
\hline Colony Characteristics & Microscopic View & Probable Mould \\
\hline $\begin{array}{c}\text { Colonies were fast growing and produced a light yellow } \\
\text { diffusible pigment after a few days incubation. }\end{array}$ & $\begin{array}{c}\text { Conidia are single-celled, hyaline to pale-brown, ovoid } \\
\text { and have smooth thin walls }\end{array}$ & $\begin{array}{c}\text { Scedosporium } \\
\text { aurantiacum }\end{array}$ \\
\hline $\begin{array}{c}\text { Colonies were flat, spreading, brown to black in colour, } \\
\text { with a dense cottony surface and a cream reverse. }\end{array}$ & $\begin{array}{c}\text { Conidiosphores were smooth-walled and bear terminal } \\
\text { verticils of metulae, each bearing phialides. Conidia } \\
\text { brown and rough-walled. }\end{array}$ & Penicillium marneffei \\
\hline $\begin{array}{c}\text { Colonies were flat spreading, brown to black in colour, } \\
\text { with a dense cottony surface and a cream reverse. }\end{array}$ & $\begin{array}{c}\text { Conidial heads are dark brown to black, radiate and } \\
\text { biserate with metulae twice as long as the phialides. } \\
\text { Conidia brown and rough-walled. }\end{array}$ & Aspergillus niger \\
\hline $\begin{array}{c}\text { Cultures were slow growing, suede-like with radial } \\
\text { furrows, initially whitish-grey and became } \\
\text { olivaceous-grey with age. }\end{array}$ & $\begin{array}{c}\text { Phialides were brown and thick walled, conidia were } \\
\text { hyaline, thin-walled, cylindrical-shaped. }\end{array}$ & Phialophora parasiticum \\
\hline
\end{tabular}

Table 5. Microscopic and Biochemical Characteristics of Yeasts from the Liquid Herbal Medications

\begin{tabular}{|c|c|c|c|c|c|c|c|c|c|c|c|}
\hline Isolates & Colour & Shape & Germ tube & Urease & Glucose & Sucrose & Maltose & Raffinose & Mannitol & Lactose & Probable yeast \\
\hline A & Cream & Round & + & - & + & - & + & - & + & - & Candida albicans \\
\hline B & Cream & Round & - & + & + & - & - & - & - & - \\
\hline
\end{tabular}

Keys:

$$
\begin{aligned}
& +=\text { positive } \\
& -=\text { negative }
\end{aligned}
$$

\begin{tabular}{|c|c|c|c|c|c|c|c|}
\hline \multicolumn{8}{|c|}{ Zones of inhibition produced by different antibiotics in the nearest millimeter (mm) } \\
\hline Bacterial isolates & $\begin{array}{c}\text { Ammoxicillin } \\
(30 \mu \mathrm{g})\end{array}$ & $\begin{array}{l}\text { Streptomycin } \\
(30 \mu \mathrm{g})\end{array}$ & $\begin{array}{l}\text { Septrin } \\
(30 \mu \mathrm{g})\end{array}$ & $\begin{array}{c}\text { Chloramphenicol } \\
(30 \mu \mathrm{g})\end{array}$ & $\begin{array}{l}\text { Ciprofloxacin } \\
(10 \mu \mathrm{g})\end{array}$ & $\begin{array}{l}\text { Sparfloxacin } \\
(10 \mu \mathrm{g})\end{array}$ & $\begin{array}{c}\text { Perfloxacin } \\
(10 \mu \mathrm{g})\end{array}$ \\
\hline E. asburiae & 15 & - & - & 17 & 22 & 10 & 24 \\
\hline E. coli & - & - & - & - & - & 15 & - \\
\hline A. baumanni & 20 & - & 17 & 15 & 20 & 16 & 20 \\
\hline Staphylococcus sp & 0 & - & 20 & 0 & 30 & 0 & 15 \\
\hline Bacillus sp & 0 & - & 20 & 0 & 19 & 0 & 12 \\
\hline
\end{tabular}

Table 6. Antibiotic sensitivity of the bacterial isolates

\begin{tabular}{|c|c|c|c|c|c|c|c|c|}
\hline \multicolumn{9}{|c|}{ Zones of inhibition produced by different antibiotics in the nearest millimeter (mm) } \\
\hline $\begin{array}{l}\text { Bacterial } \\
\text { isolates }\end{array}$ & $\begin{array}{c}\text { Gentamycin } \\
(10 \mu \mathrm{g})\end{array}$ & $\begin{array}{l}\text { Augmentin } \\
(30 \mu \mathrm{g})\end{array}$ & $\begin{array}{l}\text { Tarvid } \\
(30 \mu \mathrm{g})\end{array}$ & $\begin{array}{c}\text { Amoxil } \\
(30 \mu \mathrm{g})\end{array}$ & $\begin{array}{c}\text { Zinncef } \\
(20 \mu \mathrm{g})\end{array}$ & $\begin{array}{l}\text { Erythromycin } \\
\quad(10 \mu \mathrm{g})\end{array}$ & $\begin{array}{c}\text { Ampiclox } \\
(30 \mu \mathrm{g})\end{array}$ & $\begin{array}{l}\text { Rifampicin } \\
(25 \mu \mathrm{g})\end{array}$ \\
\hline E. asburiae & 20 & - & 18 & 0 & 0 & 0 & 0 & 0 \\
\hline E. coli & 20 & - & 15 & 0 & 0 & 0 & 0 & 0 \\
\hline A. baumanni & 20 & - & 16 & 0 & 0 & 0 & 0 & 0 \\
\hline $\begin{array}{c}\text { Staphylococcu } \\
\text { s sp }\end{array}$ & 10 & 0 & 0 & - & 20 & 10 & - & 15 \\
\hline Bacillus sp & 12 & 0 & 0 & 30 & 15 & - & - & 20 \\
\hline
\end{tabular}

Table 6. Antibiotic sensitivity of the bacterial isolates (continued)

Keys:

$$
\begin{aligned}
& -=\text { no effect } \\
& 0=\text { no test }
\end{aligned}
$$


Table 7. Antifungal Sensitivity of the Fungal Isolates

\begin{tabular}{|c|c|c|c|c|c|}
\hline \multicolumn{7}{|c|}{ Zones of inhibition produced by different antibiotics in the nearest millimeter } \\
$(\mathrm{mm})$ & Nystatin $(5 \mu \mathrm{g})$ & Ketoconazole $(10 \mu \mathrm{g})$ & Fluconazole $(10 \mu \mathrm{g})$ \\
\hline Yeast isolates & Clotrimazole $(5 \mu \mathrm{g})$ & Griseofulvin $(10 \mu \mathrm{g})$ & - & 20 & 25 \\
\hline C. albicans & 15 & - & 18 & 22 & 20 \\
\hline C. krusei & - & - & 13 & 19 & 20 \\
\hline S. aurantiacum & 12 & - & 12 & 20 & 25 \\
\hline P. marneffei & - & - & 10 & 18 & 20 \\
\hline A. niger & 20 & - & 16 & 20 & 24 \\
\hline P. parasiticum & 24 & - & & & \\
\hline
\end{tabular}

Key: $\quad$ - = no effect

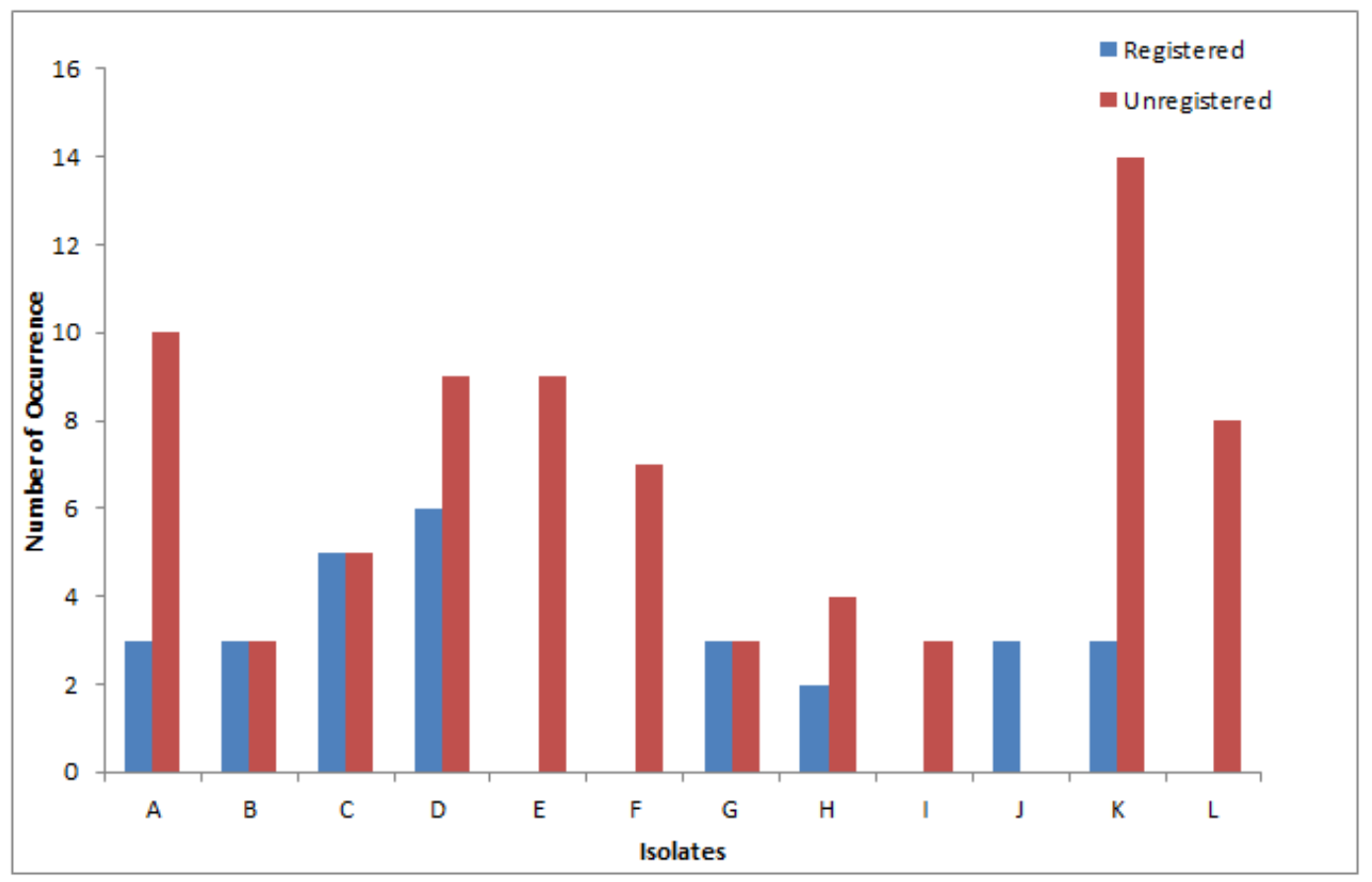

A=E. coli, B=Bacillus sp., $C=$ Staphylococcus sp., $D=E$. asburiae, $E=$ P.rettgeri, $F=$ A. baumanni, $G=P$. marneffei, $H=A$. niger, $I=S$. auranticum, $J=P$. parasiticum, $\mathrm{K}=\mathrm{C}$. albicans, $\mathrm{L}=\mathrm{C}$. krusei.

Figure 1. Frequency of Occurrence of Bacterial Isolates in the Registered and Unregistered Liquid Herbal Medications.

From the biological and molecular characterization on Table 3, 4 and 5 Providenciarettgeri, Enterobacter asburiae, Acinetobacter baumanni, Escherichia coli, Bacillus sp Staphylococcus sp, Scedosporium aurantiacum, Penicillium marneffei, Aspergillus niger, Phialophora parasiticm, Candida albicans, Candida krusei were isolated from the herbal preparation.

Antibiotic sensitivity tests results as show in table 6 for bacteria shows that the organisms were most susceptible to ciprofloxacin followed gentamycin with the least active on the organisms being streptomycin. E. asburiae was sensitive to gentamycin, perfloxacin and ciprofloxacin; E. coli was sensitive to only gentamycin; A. baumanni, was sensitive to amoxicillin, perfloxacin, amoxacillin and gentamycin; $P$. rettgeri was sensitive to amoxicillin, ciprofloxacin, gentamycin and tarivid; Stapylococcus $s p$ was sensitive to septrin, zinnacef and ciprofloxacin; Bacillus $s p$ was sensitive to amoxil, septrin and rifampicin. For the fugal isolates table
7 shows that the fugal isolates were most sensitive to fluconazole and least susceptible to griseofulvin. Candida species isolated were sensitive to ketoconazole and fluconazole only. $S$. aurantiacum was sensitive to fluconazole; $P$. marneffe $i$ was sensitive to ketoconazole and fluconazole; $A$. niger was sensitive to clotrimazole and fluconazole, $P$. parasiticum was sensitive to clotrimazole, ketoconazole and fluconazole.

The frequency of occurrence of bacterial isolates in the registered and unregistered liquid herbal medication is represented in fig. 1. From the graph it was shown that $E$. asburiae was the most frequently isolated bacteria from $30 \%$ of the registered samples while Staphylococcus sp, Bacillus $s p$ and $E$. coli followed with $25 \%, 15 \%, 15 \%$, respectively. For the unregistered samples E. coli $(25 \%)$ was the most frequently isolated followed by $E$. asburiae, $P$. rettgeri, $A$. baumanni, Staphylococcus sp and Bacillus sp. in descending order of $22.5 \%, 20 \%$ and $17.5 \%, 12.5 \%$ and $7.5 \%$ 
respectively. Fig 1 also shows the frequency of fungal isolates from all the liquid herbal medications. C. albicans $(37.5 \%)$ has highest frequency in the unregistered products with $P$. marneffei and $S$. auranticum $(7.5 \%$ each) being the lowest.

The analysis of variance between the aerobic bacterial counts in NAFDAC registered herbal medications and that of unregistered herbal medications showed significant difference between them at 5\% significance level (ANOVA). The analysis of variance between the fungal counts in NAFDAC registered herbal medications and that of unregistered herbal medications showed insignificant difference between them at 5\% significance level (ANOVA). The analysis of variance between the coliform counts in NAFDAC registered herbal medications and that of unregistered herbal medications showed a significant difference between them at 5\% significance level (ANOVA). The analysis of variance between the E.coli counts in NAFDAC registered herbal medications and unregistered herbal medications showed an insignificant difference between them at 5\% significance level (ANOVA).

\section{Discussion}

The samples were contaminated to varying degrees with bacteria and fungi which are in agreement with previous works on microbial quality by [14]. Three of the twenty NAFDAC registered herbal preparations (which were claimed to cure teeth ache, arthritis and hepatitis) were free from microbial contamination. This may be due to natural barriers and antimicrobial substances of different chemical nature as oils, peptides, liquid and organic extracts contained by certain plants which exert typical inhibitory effects on microbial growth and stability as observed by [15]. Six (30\%) had no bacterial growth; fourteen $(70 \%)$ had bacteria. Five (25\%) had no fungal growth; fifteen (75\%) had fungal growth. According to WHO standards values of the microbial limits should not exceed $10 \% / \mathrm{ml}$ for total aerobic bacteria. $10^{3} / \mathrm{ml}$ for yeast and moulds, $10^{3} / \mathrm{ml}$ for Enterobacteria whereas Salmonella and E.coli should totally be absent $[13,16] .70 \%$ of registered products met these specifications.

All the registered samples had bacteria and fungi, only about $28 \%$ of the unregistered herbal drugs complied with the WHO. This observation is similar to the observation of [17] Onyambu et al., (2013) who observed that unregulated herbal drugs were all contaminated beyond limits.

The characteristics of the isolated organisms in this study are in agreement with previous works on characterization by [18]. All the named pathogens isolated from the indigenous herbal samples in this study have been implicated in previous studies on gastroenteritis and other transmissible diseases [19].

Although Enterobacteria can be found in nature, this family possesses some indicative value towards faecal contamination. The presence of Enterobacteria and E.coli reflect the situation of faecal contamination as observed by[14]. This can be taken as an indicator for undesirable hygiene conditions. $E$. coli which contaminated $26.6 \%$ of the samples is a well-known enteropathogen and is the most common causative agent of childhood diarrhea of bacterial origin as reported by [20]. The frequency of E.coli infection has led to concern over a demand for therapeutics to treat acute E.coli infections.

Staphylococcus sp which contaminated $18.8 \%$ of the samples has been associated with a number of complications especially to immuno-compromised individuals. However contamination could provide amount of enterotoxin produced by some Staphylococcus spp, depending on the specific nature of the individual as noted by[21]Kosalec et al. (2009) according to research, diarrheal episodes of infective aetiology represent around $27 \%$ of those reported, leading to a number of serious complications and high mortality rates[22]. It can produce proteins that disable the immune system and damage tissues. It may also release exotoxins which cause gastroenteritis. The finding of other potential pathogens may also be significant.

Rajapandiyan et al. (2013)[23] also implicated Enterobacterasburiae as an opportunistic pathogen in extra-intestinal infections associated with diarrhea in children and also in nosocomial infections. Therefore, the high recovery rates of these suspected perilous bacteria from indigenous orally consumed herbal medications could be of clinical relevance.

Providencia spp are coliforms some of which cause eye and urinary tract infections in humans. The specie isolated in this study is in consonance with other works done by [6] Kunle et al. (2012). They are known to cause a number of opportunistic infections in humans and can be found in human gut. It is a major cause of traveler's diarrhea and has emerged recently as nosocomial pathogen of clinical importance. Acinetobacter baumanni is also an opportunistic pathogen and is becoming increasingly important in nosocomial infections. They are equally known to be multi-drug resistant [16].

Bacillus species are known to cause gastrointestinal infection which is characterized by diarrhea. It has been reported by [23] Rajapandiyan et al (2013) that B. subtilis is the most predominantly isolated from herbal preparation.

Three out of the bacterial isolates were coliforms. Many pathogens detected can be directly used; environmental microbiologists have generally used indicator organisms as an index of possible water contamination by human pathogens. The significance of feacal coliforms is that if these specific bacteria are present then other harmful microorganisms may also be present, such as salmonella and many more others as reported by [24].

Penicillium, Candida and Aspergillus isolated are of medical importance. Penicillium and Aspergillus are associated with food poisoning and may be responsible for infections particularly in immuno-compromised individuals moulds are responsible for biodeterioration of a number of 
substrates including raw materials and thereby decreases the medicinal potency of herbal drugs [25]. Aspergillus and Penicillium species in herbal drugs could mean that there was some growth of these organisms established before the complete drying of drugs. Since Aspergilla are capable of growing at low water content, in order to avoid such growth and the possible production of toxic metabolites, care should be taken to dry the product quickly before these molds have the chance to establish any significant growth. Members of the genera Penicillium and Aspergillus are reported to produce the widest range of mycotoxins, which with the exception of ochratoxin A and aflatoxin are patulin, citrinin, citreoviridin, griseofulvin, rubratoxin and penicillin, cyclopiazonic, secalonic or mycophenolic acids. Ochratoxin A has been reported to be nephotoxic and carcinogenic [26]. Phialophora parasiticum is a plant pathogen but it has also been reported from cases of subcutaneous infection, arthritis, mycetoma, andocarditis and mycotic keratitis [16].

The high level of microbial contamination observed in this study may be attributed to the methods of their preparation as observed by [27]. The soil, harvesting, drying, improper handling and storage conditions influence the microbial quality of herbal drugs. The presence of microbial contaminant in non-sterile pharmaceutical products can reduce or even inactivate the therapeutic activity of the products and has the potential to adversely affect patients.

Antibiotic susceptibility studies on the bacterial contamination of the liquid herbal products indicated that the isolates were susceptible to some of the antibiotics tested and resistant to others. The near uniform antibiotic susceptibility of the isolates could be attributed to their environmental origins and agrees with observation of [28] Alwakeel (2008) that some environmental bacterial isolates are sometimes susceptible to antibiotics because resistance transfer is thought to be much less efficient in the environment where microorganisms are widely separated by distance as compared with human and animal intestines [29]. Notwithstanding, E.coli was resistant to most the antibiotics used in this study and could pose a great threat to consumers as observed by [30] Ewu, 2010 who reported of the rise in the number of patients experiencing negative health consequences caused by the use of herbal medicines. The statistical analysis (ANOVA) of both registered and unregistered herbal preparations show that there is a significant difference between them in terms of microbial quality. The number of registered ones that were beyond limits is small compared to the unregistered ones.

\section{Conclusions}

This result has shown that unregulated herbal medicines marketed in Nigeria are highly contaminated with microorganisms, some of which are pathogenic compared to the regulated ones. Such unregulated medicinal products may facilitate transmission of communicable diseases in the population and therefore present a public health problem. Most of the herbal medicines analyzed in this study would be consumed at room temperature and do not get heated to above $60^{\circ} \mathrm{C}$ before consumption, thereby increasing the risk of food-borne infections.

There is consequently need to extend government regulation to herbal medicinal products to ensure that their processing, preparation or manufacture comply with Good Manufacturing Practices, and thus lessen risks to consumers and patients. The need for constant monitoring and quality control of herbal medicinal products manufactured, sold, advertised and used in Nigeria cannot be over emphasized. As herbal medicinal products are complex mixtures which originate from biological sources, great efforts are necessary to guarantee a constant adequate quality. By carefully selecting the plant material and a standardized manufacturing process, the pattern and concentration of constituents of herbal medicinal products should be kept as constant as possible as this is a prerequisite for reproducible therapeutic results. Quality has to be built into the whole process beginning from the selection of propagation material to the final product reaching the consumer.

\section{REFERENCES}

[1] Ampofo, J. A., Tetteh, W. and Bello, M. (2012). Microbiological Profile of Some Ghanian Herbal Preparations-Safety Issues and Implications for the Health Professions. Open Journal Of Medical Microbiology, 2:121-130.

[2] Abel, C. and Busia, K. (2005). An Exploration Ethnobotanical Study of the Practice of Herbal Medicine by the Akan People of Ghana. Alternative Medicine Review.6:70-77.

[3] WHO, (2003). WHO Guidelines on Good Agricultural and Collection Practices. [GCAP] For Medicinal plants. Geneva. WORLD HEALTH organization. pp: 167-175.

[4] Oktem, M., Eroglu, D., Karahan, H. B., Taskintuna, N., Kuscu, E. and Zeyneloglu, H. B. (2007).Drugs Hawked in Minna, Niger State. Journal of Pharmacology and Resolution. 6:661-670.

[5] Kigen, G. K., Hillary, K., Ronoh, B. C., Wilson, K., Kipkore, D. and Joseph, K. R. (2013). Current Trends of Traditional Herbal Medicine Practice in Kenya: A Review. African Journal of Pharmacology and Therapeutics.2:32-37.

[6] Kunle, O. F., Egharevba, H. O. and Ahmadu, P.O. (2012). Standardization of Herbal Medicines - A Review. International Journal of Biodiversity and Conservation.4:101112.

[7] Chitrarekha, K., Adwait, D. and Shridhar, M. (2010). Assessment of Microbial Contamination in Commercial Herbal Oral Medicinal Liquids. International Journal of Pharma Research and Development. 2(9):0974-9446.

[8] Mendes, E., Herdeiro, M. T. and Pimentel, F. (2010). The Use of Herbal Medicine Therapies by Cancer Patients. ActaMédica 
Portuguesa. 23(5):901-908.

[9] Okunlola, A., Adewoyin, B. A. and Odeku, A. O. (2007). Evaluation of Pharmaceuticals and Microbial Qualities of Some Herbal Medicinal Products in South Western Nigeria. Tropical Journal of Pharmacology and Resolution. 6:661-670.

[10] Clinical and Laboratory Standard Institute (2002a). Reference Method for Broth Dilution Antifungal Susceptibility Testing Yeast; Approved Standard-Second edition. CLSI Document M27-A2 [ISBN 1-56238-469-4]. CLSI, Pennsylvania, USA. PP; $23-50$

[11] Clinical and Laboratory Standard Institute (2002b). Reference Method for Broth Dilution Antifungal Susceptibility Testing Yeast; Approved Standard-Second Edition. CLSI Document M27-A2 [ISBN 1-56238-470-8]. CLSI, Pennsylvania, USA. PP; $12-35$.

[12] Clinical and Laboratory Standard Institute (2006). Method for Antifungal Disk Susceptibility Testing Yeast; Approved Guidelines. CLSI DocumentM44-A2 [ISBN 1-56238-488-0]. CLSI, Pennsylvania, USA. PP; 67-90.

[13] WHO (2007). Guidelined for Assessing Quality of Herbal Medicines with Reference to Contaminants and Residues, $3^{\text {rd }}$ ed. Vol. 1. Recommendations. Geneva, World Health Organization. Pp: 130-143.

[14] Czech, E., Kneifel, W. and Kopp, B. (2001). Microbiological Status of Commercially Available Medicinal Herbal Drugs- A Screening Study. PlantaMedica. 67: 263-269.

[15] Twari, B. K., Valdramidi, V. P., O'donnell. C. P., Muthukumarappan, K., Bourke, P. and Cullen, P. J. (2009). Application of Natural Antimicrobials for Food Preservation. Journal of Agricultural and Food Chemistry.57:5987-6000.

[16] Idu, M., Erhabor, J. O. and Idele, S. O. (2011). Microbial Load of Some Medicinal Plants Sold in Local Markets of Benin City, Nigeria. International Journal of Medicinal and Aromatic Plants. 1(3):272-277.

[17] Onyambu, M. O., Chepkwony, H. K., Thoithi, G. N., Ouya, G. O. and Osanjo, G. O. (2013). Microbial Quality of Unregulated Herbal Medicinal Products in Kenya. African Journal of Pharmacology and Therapeutics. 2(3): 70-77.

[18] Willey, J. M., Sherwood, L. M. and Woolverton, C. J. (2008). Isolation of Pure Bacterial Cultures from Specimens. In: Prescott, Harley and Klein's Microbiology $\left(7^{7^{\text {th }}} \mathrm{ed}\right)$. Mcgraw-Hill, New York. Pp 714-796.
[19] Pearce, M. C., Fenlon, D., Low, J. C., Smith, A. W., Knight, H. I., Evan, J., Foster, G., Synge, B.and Gunn, G. J. (2004). Distribution of Esherichia coli 0157 in Bovine Faecal Parts and Its Impact on Estimates of the Prevalence of Faecal Shedding. Applied Environmental Microbiology. 70: 5737-5743.

[20] Bonkoungou, I. J., Haukka, K., Osterblad, M., Hakanen, A. J., Traore, A. S., Barro, N. and Siitonen, A. (2013). Bacterial and Viral Eiology of Childhood Diarrhea in Ouagadougou, Burkina Faso. BMC Paediatrics. 13:36.

[21] Kosalec, I., Cvek, J. and Kopp, B. (2002). Contamination of Medicinal Herbs and Herbal Products. Archives of Industrial Hygiene and Toxicology, 60:485-501.

[22] Wylie, J. L. and Norwick, D. L. (2005). Molecular Epidemiology of Community and Health Care-Associated Methicillin Resistant Staphylococcus aureus in Manitoba, Canada. Journal of Clinical Microbiology. 43(6):2830-2836.

[23] Rajapandiyan, K., Shanthi, S. and Vidya, S. (2013). Assessment of Microbial Quality in Marketed Herbal Drugs Sold in Trichy City. International Journal of Pharmaceutical, Chemical and Biological Sciences. 3(3):894-898.

[24] Forest, J. (2004). Feacal Coliforms. University Of Iowa Hygienic Laboratory Manual. 36(2):4.

[25] Kumar, A., Shukla, R., Singh, P. and Dubey, N. K. (2009). Biodeterioriation of Some Herbal Raw Materials by Storage Fungi and Aflatoxin and Assessment of Cymbopogon flexuosus Essential Oil and Its Components as Antifungal. International Journal of Food Microbiology. 49:75-83.

[26] Busse, W. (2000). The Significance of Quality for Efficacy and Safety of Herbal Medicinal Products. Drug Information Journal. 34:15-23.

[27] Rocha, R. P., Melo, E. C. and Radünz, L. L. (2011). Influence of Drying Process on the Quality of Medicinal Plants: A review. Journal of Medicinal Plants Research. 5(33):7076-7084.

[28] Alwakeel, S. S. (2008). Microbial and Heavy Metal Contamination of Herbal Medicines. Research Journal of Microbiology. 3(12): 683-691.

[29] Amaike, S. and Keller, N. P. (2011). Aspergilus flavus. Annual Review of Phytopathology.49: 107- 133.

[30] Ewu, I. (2010). The Role of NAFDAC in Regulation and Control of Herbal Medicines in Nigeria. Journal of Pax and Herbal Magazine. 3:23-30. 